\title{
Potential Role of Glucose Transporters in Glucose Homeostasis: a Narrative Review
}

\author{
Muslim Bin Aqeel', Sana Ashiq ${ }^{2, *}$, Mayyda Asif Bajwa ${ }^{3}$, Kanwal Ashiq ${ }^{3}$ \\ ${ }^{1}$ NUR International University Lahore-Pakistan. \\ ${ }^{2}$ Centre for Applied Molecular Biology, University of the Punjab, Lahore-Pakistan. \\ ${ }^{3}$ Faculty of pharmaceutical Sciences, Superior University Lahore-Pakistan.
}

\begin{abstract}
Authors' Contributions
1, 3 \& 4 Data Collection \& Processing, Data

Analysis and/or Interpretation, Drafting of

Manuscript, Critical Review.

2 Conception \& Study design, Data Analysis and/or Interpretation, Drafting of Manuscript, Critical Review.
\end{abstract}

\section{Acknowledgement}

Article info.

Received: August 16, 2020

Accepted: May 26, 2021

Funding Source: Nil

Conflict of Interest: Nil

Cite this article: Aqeel MB, Ashiq S Bajwa MA, Ashiq K. Potential Role of Glucose Transporters in Glucose Homeostasis: a Narrative Review. RADS J Pharm Pharm Sci. 2021; 9(1):84-87.

*Address of Correspondence Author: sanaashiq72@gmail.com

\begin{abstract}
A B S T R A C T
Background: The GLUT2 (glucose transporter 2) is a glucose sensitive receptor gene found in the liver, pancreas, and sometimes in the CNS. The experiments conducted on the genetically modified mice revealed their role in the different regulatory mechanisms.
\end{abstract}

Objective: The current narrative review aims to elucidate the role of GLUT2 in glucose homeostasis.

Methods: The recent related articles were reviewed with the help of different databases including PubMed, Google Scholar, Springer link, and Science direct. To ensure the credibility of data, articles published only in indexed journals were considered.

Results: In the liver (hepatocytes), it's reported that there is an unsuspected glucose pathway (output) that is dependent on the membrane mechanism is in progress. In humans, due to the mutation in the GLUT2 gene, there is a syndrome known as "Fanconi-Bickel syndrome" which dictates a glycogen storage disease type 11 disease characterized by kidney and liver disorders.

Conclusion: It is concluded from the genome-wide association studies that the genetic mutations in GLUT2 result in an increased risk of high cholesterol, cardiovascular diseases (CVDs), and fasting hyperglycemia.

Keywords: GLUT2, Fanconi-Bickel syndrome, CVDs, Glucose Transporter.

\section{INTRODUCTION}

Glucose transporter 2 (GLUT2), famously known as solute carrier family 2 (cellular glucose helper), member 2 (SLC2A2). The SLC2A2 is approximate $30 \mathrm{~kb}$ in size. This gene consists of ten introns and eleven exons. It stands as a transmembrane protein possessing the function of cellular glucose transport in cell membranes. The major contributor to this is the movement of blood glucose between the liver and blood. The gene is found amongst humans, where the respective protein is found encoded by the SLC2A2 gene [1]. It is to note that the error in this gene is linked with a typical kind of glycogen storage disease (GSD) i.e. Fanconi Bickel syndrome (FBS) [2].

In SLC2A2 knockout mice suppress glucose stimulated insulin secretion and inhibit beta-cell glucose uptake, and the transgenic GLUT2 gene has been shown to protect against a variety of disorders in the mice. However, while the lack of GLUT2 adaptation is not well established [3], it is important to remember that the major cause of non-diabetes is likely to result in infants older than average. Large may be a good measure of good glycemic control and healthy GLUT2 status. Establish the onset of blood sugar attacks between the blood vessels and the space within the kidney. GLUT2 has been seen 
playing a major part in osmoregulation, and prevents edema development, transient ischemic stroke especially when blood glucose levels are above average [4]. GLUT2 may be referred to as "diabetic glucose transporter" or "stress hyperglycemia glucose transporter" [5].

Fanconi Bickel syndrome is a glycogen storage disorder caused by autosomal recessive pathways and also due to conformational polymorphisms in the SLC2A2 gene causing to loss of GLUT2 transporter expression. It is accepted as a rarely occurring disease owing to approximately 100 reported so far as seen through been in the literature review. SLC2A2 gene is used for GLUT2, which is also a lowaffinity transport protein that is involved in sugarinduced homeostasis, for instance as in pancreatic $\beta$ cells, hepatocytes, and the tubular cells. The FBS has a marked reduction in galactose and glucose utilization, hepato-renal glycogen accumulation and tubular nephropathy. GLUT2 deficiency and hypotension result in postprandial hyperglycemia, fasting hypoglycemia, hepatomegaly, diabetes mellitus (DM) galactose, and glucose deficiency, probable defiance against glucagon and adrenaline, malignant growths, and rickets. The part of GLUT2 shortage in the origin of DM is not completely established [6].

Many mutations related to the GLUT2 gene are well interpreted for FBS [7], to date, forty-six polymorphisms (missense, splice site variants, deletions, insertions, and nonsense) were identified in the SLC2A2 gene which has a strong association with the Fanconi Bickel syndrome. Further research in FBS patients confirmed that a person must possess 2 pathogenic SLC2A2 mutation with homozygous or compound heterozygous for the manifestation of the disease. Figure 1 explains the genetic polymorphisms in the GLUT2 gene [8].

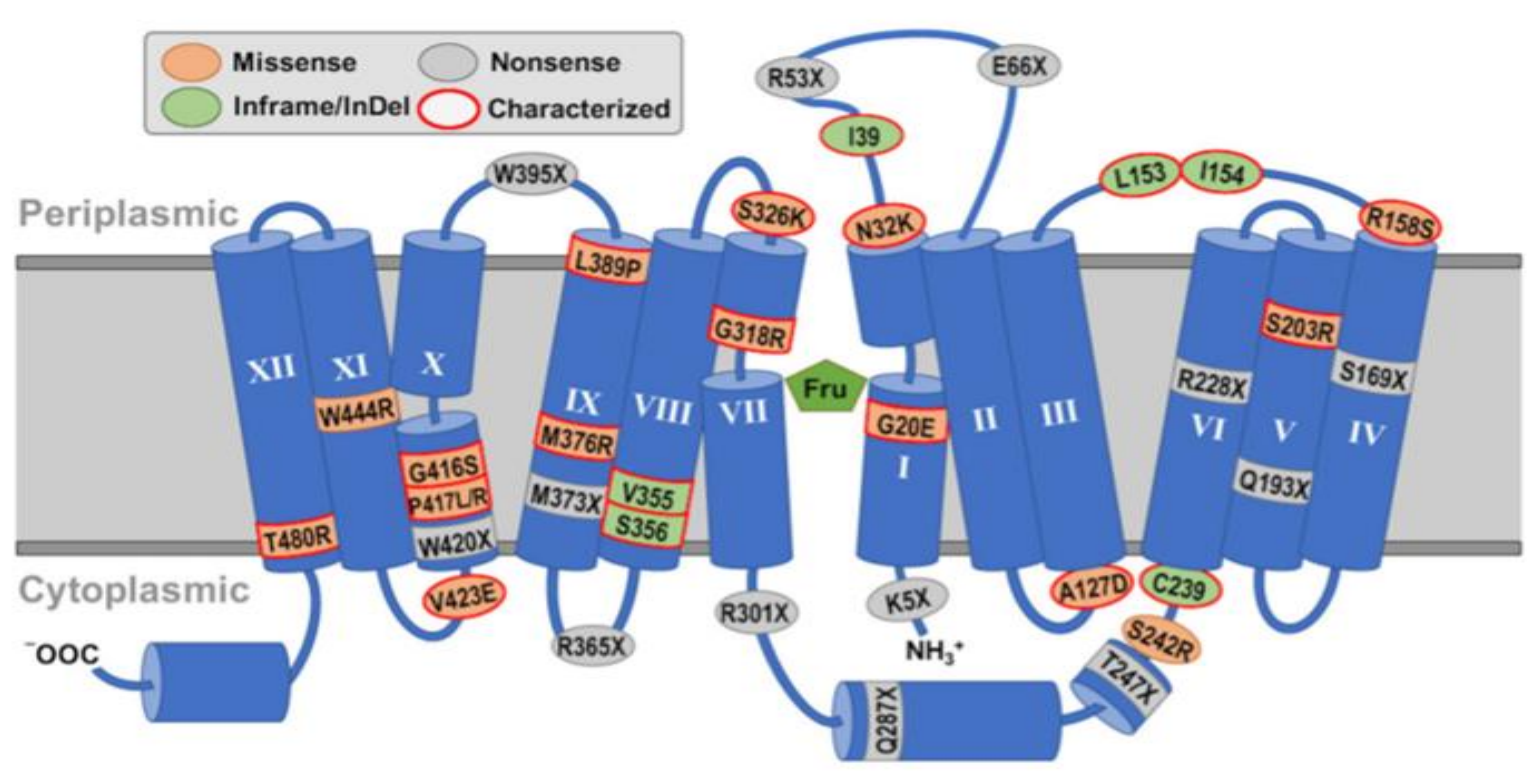

Figure 1. Mutation including missense, non-sense, inframe /Del and characterized within the GLUT2 transporter which are associated with FBS. Roman numerals I-XII indicate GLUT2 transmembrane helix, red border for functional characterization and "Fru" represents the fructose [8].

\section{Glucose Transporter (GLUT2)}

It was first cloned in 1988 from human liver cDNA libraries and rats. These glucose transporter 1 showed $55 \%$ similarity and $80 \%$ homology with the glucose transporters 1 . It is composed of five hundred and twenty-four amino acids. It is mainly present in the liver, where it plays an essential role in the transportation of hexose, intestine, kidney, and islet cells [9]. It consists of fourteen isoforms. It has a high affinity for glucosamine and a low affinity for glucose, fructose, mannose, and galactose. It has a key role in the transepithelial glucose transport in the kidney [10].

\section{Liver Glucose Sensing and GLUT2}

The hepatoportal supply is mainly regulated by the afferents [11], which operate after glucose is activated by the shield made by the hepatoportal sensor, 
requiring GLUT2 expression, and was further regulated by a flow of 5 ' AMP-stimulated protein kinase [12]. It has been shown that in situ, the regulation of the first degree of insulin depends on a physiological signal that glucose is entering the hepatoportal artery, and may have been transmitted leading to neural beta cells.

\section{Glucose Transport Mechanism}

It is known that glucose travels into the enterocyte making way from the brush border membrane in the intestine via sodium glucose cotransporter 1 (SGLT1). It thereby is discharged all over the basolateral membrane, traveling down the concentration gradients through utilizing the GLUT2. However, $\mathrm{Na}+/ \mathrm{K}+$ pump, found in the basolateral membrane plays a major part in the maintenance of the applicable gradient of SGLT1. It is also noted that fructose is also transferred from the brush border membrane and forced out bilaterally. This is accomplished by the well-known mechanism of facilitated diffusion by way of GLUT5.

\section{Fanconi-Bickel Syndrome}

It is a state depicting the sparse occurrence of glycogen storage disease (GSD). As a result of heterozygous or homozygous mutations in GLUT2, the gene further promotes the interaction of pancreatic b-cells, enterocytes, hepatocytes, and other human cells. Most patients have a common form of chronic disease: hepatomegaly seconds with glycogen build-up, galactose and sugar imbalance, fasting hypoglycemia, distinct tubular nephropathy, and severe tumor growth. The efficacy of GLUT2 in DM is still debated because it is not the major cause of glucose tolerance in pancreatic cells in humans. $[13,14]$.

FBS results as mutations in SLC2A2 including eleven exons up to $30-\mathrm{kb}$ region on chromosome 3 (3q26.126) [15]. The long-acting transporter 2 (GLUT2 or GTR2) proteins, that are highly implied in enterocytes, hepatocytes, pancreatic $\beta$-cells, stem, and neural cells [16]. GLUT2 functions as a glucose sensor, monosaccharide transporter, and plasma membrane sugar that stimulates homeostatic sugar in the body [17]. Until now, greater than $80 S L C 2 A 2$ mutations are noted to cause FBS at one time worldwide.

Amongst many pathways with $S L C 2 A 2$, the gene used for circulating glucose, GLUT2, has been linked to FBS, is also rare with elevated glycogen content, and also reported in the 100 published articles. These deficiencies rarely reduce GLUT2 transporter activity, causing significant downstream effects. To date, 46 SLC2A2 mutations are found to be associated with Fanconi Bickel syndrome. FBS is a dominant disease; autosomal and for FBS to be expressed in humans, it must be caused by homozygous or heterozygous cells and two pathogenic SLC2A2 mutations.

In addition to people who are at risk of GLUT2-related FBS events, those with less than one are small (medium frequency between $1 \%$ to $5 \%$ ) or normal (low injection> 5\%). The SLC2A2 allele may be at stake for several metabolic characteristics. For instance, a genome-wide sequence analysis (GWAS) has specified the most widespread genes in SLC2A2 that are associated with a heightened risk of type 2 diabetes [18].

\section{Genome-Wide Association Studies}

GLUT 2 is responsible to regulate impairment glucose (fasting) for the sake of risk of type 2 diabetes Mellitus and Heart Attack. The only transporter required for glucose homeostasis and its metabolism is GLUT2. Genome-wide studies have reported that GLUT2 variants are associated with fasting hyperglycemia and enhanced risk of type 2 diabetes in humans, the association of GLUT2 mutation with diabetes in infant, high-risk, and potentially fatal, type 2 diabetes may be best described as a glucose disorder and may cause loss of first-phase insulin resistance, immune control, and progression forward development of the cream is not acceptable.

In conclusion, in humans, several polymorphisms in this gene showed to be associated with the FanconiBickel syndrome, which is well known for being an autosomal recessive disorder as seen in the metabolism of carbohydrates. The GLUT2 inactivation in mice due to homologous recombination incites drastic glucosuria. Certainly, it turned out as per the report that GLUT2 cell surface expression influences glucose induced insulin secretion. GLUT2 also possesses the ability to allow a consistent message to the entire body via signals sent out to both sugar scarcity and abundance. Moreover, novel opportunities for the re-evaluation of GLUT2 in obesity, diabetes, and insulin resistance may help in the future prevention of the diseases.

\section{REFERENCES}

1. Demirbilek H, Galcheva S, Vuralli D, Al-Khawaga $\mathrm{S}$, Hussain K. Ion transporters, channelopathies, and glucose disorders. Int $\mathrm{J}$ Mol Sci. 
2019;20(10):2590.

DOI: https://doi.org/10.3390/ijms20102590.

2. Sharari S, Abou-Alloul M, Hussain K, Ahmad Khan F. Fanconi-Bickel Syndrome: a review of the mechanisms that lead to dysglycaemia. Int $\mathrm{J}$ Mol Sci. 2020;21(17):6286.DOI: https://doi.org/10.3390/ijms21176286.

3. Mueckler M, Thorens B. The SLC2 (GLUT) family of membrane transporters. Mol Aspects Med. 2013;34(2-3):121-38. DOI: https://doi.org/10.1016/j.mam.2012.07.001.

4. Stolarczyk E, Le Gall M, Even P, Houllier A, Serradas $\mathrm{P}$, Brot-Laroche $\mathrm{E}$, et al. Loss of sugar detection by GLUT2 affects glucose homeostasis in mice. PLoS One. 2007;2:e1288. DOI: https://doi.org/10.1371/journal.pone.0001288.

5. Panchal NK, Jerine Peter S, Chhetri P, Sil C, Sabina AF. In-silico docking analysis of the active compounds of Murraya koenigii seeds with the modelled protein of unstructured Diabetes genes. Res J Pharm Technol. 2020;13(12):6163-9.

6. Sharari S, Aouida M, Khan F, Al-Khawaga S, Mohammed I, Haris B, et al. A novel SLC2A2 mutation implicated in Fanconi Bickel syndrome and dysglycemia. In58th Annual ESPE 2019 Aug 22 (Vol. 92). European Society for Paediatric Endocrinology.

7. Sansbury FH, Flanagan SE, Houghton JA, Shen FS, Al-Senani AM, Habeb AM, et al. SLC2A2 mutations can cause neonatal diabetes, suggesting GLUT2 may have a role in human insulin secretion. Diabetologia. 2012;55(9):2381-5. DOI: https://doi.org/10.1007/s00125-012-2595-0.

8. Enogieru OJ, Ung PM, Yee SW, Schlessinger A, Giacomini KM. Functional and structural analysis of rare SLC2A2 variants associated with Fanconi-Bickel syndrome and metabolic traits. Hum Mutat. 2019;40(7):983-95. DOI: https://doi.org/10.1002/humu.23758.

9. Long W, Cheeseman $\mathrm{Cl}$. Structure of, and functional insight into the GLUT family of membrane transporters. Cell Health Cytoskelet. 2015;7:167. DOI: 10.2147/CHC.S60484.

10. Thorens B. GLUT2, glucose sensing and glucose homeostasis. Diabetologia. 2015;58(2):221-32. DOI: https://doi.org/10.1007/s00125-014-3451-1.
11. Jensen KJ, Alpini G, Glaser S. Hepatic nervous system and neurobiology of the liver. Compr Physiol. 2013;3(2):655-65. DOI: https://doi.org/10.1002/cphy.c120018.

12. Kang $\mathrm{OH}$, Shon MY, Kong $R$, Seo YS, Zhou T, Kim DY, et al. Anti-diabetic effect of black ginseng extract by augmentation of AMPK protein activity and upregulation of GLUT2 and GLUT4 expression in $\mathrm{db} / \mathrm{db}$ mice. BMC Complement Altern Med. 2017;17(1):341. DOI: https://doi.org/10.1186/s12906-017-1839-4.

13. Batool H, Zubaida B, Hashmi MA, Naeem M. Genetic testing of two Pakistani patients affected with rare autosomal recessive Fanconi-Bickel syndrome and identification of a novel SLC2A2 splice site variant. J Pediatr Endocrinol Metab. 2019;32(11):1229-33.

DOI: https://doi.org/10.1515/jpem-2019-0235.

14. Şeker-Yılmaz B, Kör D, Derya Bulut $F$, Yüksel B, Karabay-Bayazıt A, Kemal Topaloğlu A, et al. Impaired glucose tolerance in Fanconi-Bickel syndrome: eight patients with two novel mutations. Turk J Pediatr. 2017;59(4). DOI: 10.24953/turkjped.2017.04.010.

15. Gupta N, Nambam B, Weinstein DA, Shoemaker LR. Late Diagnosis of Fanconi-Bickel Syndrome: Challenges With the Diagnosis and Literature Review. JIEMS. 2016;4. DOI: http://dx.doi.org/10.1177/2326409816679430.

16. Barahona MJ, Llanos $P$, Recabal $A$, Escobar-Acuña K, Elizondo-Vega R, Salgado M, et al. Glial hypothalamic inhibition of GLUT2 expression alters satiety, impacting eating behavior. Glia. 2018 Mar;66(3):592-605. DOI: 10.1002/glia.23267.

17. Leturque A, Brot-Laroche E, Le Gall M. GLUT2 mutations, translocation, and receptor function in diet sugar managing. Am $\mathrm{J}$ Physiol Endocrinol Metab. 2009;296:E985-92. DOI: https://doi.org/10.1152/ajpendo.00004.2009.

18. Dupuis J, Langenberg C, Prokopenko I, Saxena R, Soranzo N, Jackson AU, et al. New genetic loci implicated in fasting glucose homeostasis and their impact on type 2 diabetes risk. Nat Genet. 2010;42:105-16.

DOI: https://doi.org/10.1038/ng.520. 